\title{
Differential Cardiovascular Effects of Endotoxin Derived from Escherichia coli or Pseudomonas aeruginosa
}

\author{
Aya MATSUSHITA' ${ }^{1)}$, Mitsunori IWASE ${ }^{1,2)}$, Yosuke KATO'), \\ Sahoko ICHIHARA ${ }^{3)}$, Gaku ICHIHARA ${ }^{4)}$, Hirotaka KIMATA ${ }^{1)}$, Keiko HAYASHI ${ }^{1}$, \\ Katsunori HASHIMOTO ${ }^{1)}$, Toyoharu YOKOI ${ }^{1}$, Akiko NODA ${ }^{1)}$, Yasuo KOIKE'), \\ Mitsuhiro YOKOTA ${ }^{5)}$, and Kohzo NAGATA ${ }^{1)}$
}

\begin{abstract}
1)Pathophysiological Laboratory Sciences, Graduate School of Medicine, Nagoya University, 1-1-20 Daiko-minami, Higashi-ku, Nagoya 461-8673, 2)Division of Integrated Medicine, Toyota Memorial Hospital, 1-1 Heiwa-cho, Toyota 471-8513, 3)Department of Human Functional Genomics, Life Science Research Center, Mie University, 1577 Kurimamachiya-cho, Tsu 514-8507, ${ }^{4}$ Department of Occupational and Environmental Health, Graduate School of Medicine, Nagoya University, 65 Tsurumai-cho, Showa-ku, Nagoya 466-8550, and ${ }^{5)}$ Department of Genome Science, School of Dentistry, Aichi-Gakuin University, 1-100 Kusumoto-cho, Chikusa-ku, Nagoya 464-8650, Japan
\end{abstract}

\begin{abstract}
Sepsis is characterized by various symptoms, signs and underlying pathophysiology. To investigate possible mechanisms underlying this diversity, we compared the cardiovascular effects of lipopolysaccharide (LPS) derived from Escherichia coli (E-LPS) with those of LPS from Pseudomonas aeruginosa (P-LPS) in rats. We also examined the possible roles of tumor necrosis factor- $\alpha$ (TNF- $\alpha$ ) and oxidative stress in LPSinduced cardiovascular damage. E-LPS (10 mg/kg body weight) or P-LPS (2 mg/kg body weight) was administered intravenously to Wistar rats. Echocardiography was serially performed. E-LPS induced an increase in left ventricular fractional shortening that persisted for at least $6 \mathrm{~h}$, whereas $P$-LPS elicited an initial increase and a subsequent decrease in this parameter. Histological analysis revealed that P-LPS induced interstitial edema, congestion, intramyocardial bleeding, myocardial necrosis, infiltration of inflammatory cells, and formation of fibrin thrombi in the heart, whereas no pathological changes were apparent in the hearts of rats treated with E-LPS. Furthermore, the plasma concentration of TNF- $\alpha$ in rats treated with $P$-LPS was greater than that in rats treated with E-LPS, but the glutathione redox ratio in the heart was not affected by either type of LPS. In conclusion, E-LPS and $P$ LPS induced distinct patterns of functional and structural responses in the cardiovascular systems of rats. These differential responses may be attributable in part to the difference in the associated increases in the plasma concentration of TNF- $\alpha$. The cardiovascular effects of LPS thus depend on the causative organisms.
\end{abstract}

Key words: echocardiography, endotoxin, lipopolysaccharide, sepsis, tumor necrosis factor- $\alpha$ 


\section{Introduction}

Sepsis is a major cause of human mortality after surgery, with death resulting largely from the effects of endotoxin released from live or dead bacteria [6]. Sepsis is characterized by various symptoms and signs including fever, fatigue, loss of appetite, mental difficulties, increased or decreased cardiac output, hypotension, as well as injury to the vascular endothelium and intestinal epithelium [29]. Clinical studies have characterized the pathophysiology and potential mechanisms of cardiovascular deterioration during septic shock in humans [22, 23]. Endotoxin, or lipopolysaccharide (LPS), produced by Gram-negative bacteria stimulates monocytes and macrophages to release various cytokines including tumor necrosis factor- $\alpha$ (TNF- $\alpha$ ), which contributes to organ dysfunction in sepsis [20]. TNF- $\alpha$ has been shown to increase both the expression of the inducible isoform of nitric oxide (NO) synthase and the generation of reactive oxygen species in vascular smooth muscle cells. It also has a direct negative inotropic effect on cardiac myocytes, as well as inducing apoptosis and ventricular remodeling in the heart, the latter action being manifest as increased production and accumulation of collagen within the injured myocardium [4]. Reactive oxygen or nitrogen species also impair substrate metabolism and energy production in the heart [5]. Sepsis is thus accompanied by a series of events, including cytokine production, generation of oxidative stress, and impairment of metabolism, that lead to myocardial dysfunction and death.

Studies of the effects of LPS-induced sepsis on the cardiovascular system have been performed in a variety of species, especially in rats [2, 31]. However, serial measurements of cardiovascular parameters have been limited mostly to either blood pressure or heart rate in rodent models. Echocardiographic techniques have been applied successfully in rodents [11-15, 21]. With the use of echocardiography, we have previously shown that platelet-activating factor plays an important role in the changes in cardiac structure and function induced by endotoxin derived from Klebsiella pneumoniae [13]. Our preliminary observations have indicated that the cardiovascular effects of LPS derived from Escherichia coli (E-LPS) or Pseudomonas aeruginosa (P-LPS) differed from those of LPS from
K. pneumoniae, suggesting that the nature of LPS-induced cardiovascular alterations might depend on the bacterial species responsible for infection and sepsis. In this study, we applied echocardiography and other approaches to compare the cardiovascular effects of ELPS with those of P-LPS. Furthermore, given that myocardial oxidative stress has been implicated in ischemia-reperfusion injury as well as in the development of heart failure [26], we also examined the possible role of such stress in the cardiovascular effects of LPS.

\section{Materials and Methods}

\section{Animals}

Male Wistar rats were obtained from Japan SLC (Hamamatsu, Japan) and housed in an animal facility at 22 to $24^{\circ} \mathrm{C}$ with a 12 -h light, 12 -h dark cycle. They had free access to food (CE-2, CLEA Japan, Tokyo, Japan) and water. The experimental protocol was approved by the Committee on Laboratory Animal Utilization of Nagoya University, and the animals were handled in accordance with the guidelines of Nagoya University Graduate School of Medicine as well as with the Guide for the Care and Use of Laboratory Animals (National Institutes of Health, Bethesda, MD).

\section{Physiological measurements}

Rats at 8 weeks of age were weighed (body weight, $244.8 \pm 7.1 \mathrm{~g}$ ), anesthetized with an intraperitoneal injection of sodium pentobarbital ( $50 \mathrm{mg} / \mathrm{kg}$ body weight), and allowed to breathe spontaneously. Supplemental doses of anesthetic were administered to maintain a uniform level of anesthesia throughout the experiment. A polyethylene catheter (SP 45, SP 10, Natsume, Tokyo, Japan) filled with $0.9 \%$ saline was inserted into the right carotid artery for measurement of arterial pressure and sampling of blood. The catheter was connected to a pressure transducer (CDX, Fukuda, Tokyo, Japan). The right jugular vein was also catheterized for the administration of E-LPS, P-LPS, or physiological saline. Electrocardiographic leads were attached to each limb with the use of needle electrodes (IMR-110520, Inter Medical, Nagoya, Japan). Mean and phasic arterial pressures and electrocardiograms were monitored continuously with the use of a multichannel polygraph system (MCS-5000, Fukuda, Tokyo, Japan) at a paper speed of $100 \mathrm{~mm} / \mathrm{s}$. Systolic, diastolic, and mean arterial pressures were determined by averaging data ob- 
tained from more than five consecutive cycles. After both arterial pressure and heart rate had stabilized, E-LPS $(n=6)$, P-LPS $(n=6)$, or physiological saline $(n=4)$ was administered over $30 \mathrm{~s}$ with a syringe pump (TE-311, Terumo, Tokyo, Japan). E-LPS (serotype O55:B5, Sigma, St. Louis, MO) was administered at a dose of $10 \mathrm{mg} / \mathrm{kg}$ in a volume of $0.1 \mathrm{ml}$ per $100 \mathrm{~g}$ of body weight, which is the minimum dose that induced significant changes in blood pressure and cardiac function as assessed by echocardiography in preliminary experiments. P-LPS (serotype 10 , Sigma, St. Louis, MO) was administered at a dose of $2 \mathrm{mg} / \mathrm{kg}$ in a volume of $0.1 \mathrm{ml}$ per $100 \mathrm{~g}$ of body weight, which is the same dose of LPS derived from $K$. pneumoniae that was used in our previous study [13].

Echocardiography was performed with a 13-MHz transducer (Nemio, Toshiba Medical, Tokyo, Japan). Left ventricular (LV) end-diastolic internal dimension (LVDd), LV end-systolic internal dimension (LVDs), intraventricular septal wall thickness (IVST), and LV posterior wall thickness (LVPWT) were measured in M-mode recordings at the papillary muscle level in the LV short-axis view, and LV fractional shortening (LVFS) was calculated from the equation: LVFS $(\%)=[(\mathrm{LVDd}-\mathrm{LVDs}) /$ LVDd] $\times 100$. Echocardiographic recordings were performed before and after the administration of LPS.

\section{Assessment of oxidative stress}

Rats at 8 weeks of age (body weight, $245.8 \pm 3.4 \mathrm{~g}$ ) were anesthetized with an intraperitoneal injection of sodium pentobarbital $(50 \mathrm{mg} / \mathrm{kg})$ and were sacrificed at 1,4 , or $7 \mathrm{~h}$ after administration of E-LPS $(\mathrm{n}=12)$, PLPS $(n=12)$, or physiological saline $(n=12)$. The heart and liver were removed, immediately frozen in liquid nitrogen, and subsequently homogenized in $0.5 \mathrm{ml}$ of a solution containing $10 \mathrm{mM}$ HEPES ( $\mathrm{pH}$ 7.4), $320 \mathrm{mM}$ sucrose, and $10 \mathrm{mM}$ EDTA. The homogenate was centrifuged at $100,000 \times \mathrm{g}$ for $1 \mathrm{~h}$ at $4^{\circ} \mathrm{C}$, and the resulting supernatant was collected both for determination of the amounts of reduced and oxidized glutathione and for measurement of protein concentration with an assay kit (Bio-Rad, Hercules, CA) and bovine serum albumin as standard. Total glutathione was determined with a recycling assay based on glutathione reductase and 5,5'-dithiobis-(2-nitrobenzoic acid) as previously described [28], and oxidized glutathione was also assayed as described [8].

\section{Determination of the plasma concentration of $c \operatorname{Tn} T$}

Arterial blood samples $(0.6 \mathrm{ml})$ were collected both before and 1, 4, and $7 \mathrm{~h}$ after administration of E-LPS $(n=6)$, P-LPS $(n=6)$, or physiological saline $(n=4)$. The plasma cardiac troponin $\mathrm{T}$ (cTnT) level was measured with an electrochemiluminescence immunoassay [16].

\section{Determination of the plasma concentration of TNF- $\alpha$}

Arterial blood samples $(0.6 \mathrm{ml})$ were collected both before and $0.5,1,1.5,2,3$, and $4 \mathrm{~h}$ after administration of E-LPS (n=5), P-LPS ( $n=7)$, or physiological saline $(n=4)$. The plasma concentration of TNF- $\alpha$ was measured by an enzyme-linked immunosorbent assay kit (Pierce, Rockford, IL).

\section{Pathology}

After the echocardiographic experiments, the heart and kidneys were removed from rats and fixed by immersion in 20\% phosphate-buffered formalin. The fixed tissue was dehydrated, embedded in paraffin, sectioned at a thickness of $4 \mu \mathrm{m}$, and stained with hematoxylineosin or phosphotungstic acid-hematoxylin.

\section{Statistical analysis}

Data are presented as means \pm SEM. Differences among the groups were evaluated by analysis of variance followed by Scheffe's test. A $P$ value of $<0.05$ was considered statistically significant.

\section{Results}

\section{Arterial pressure and echocardiographic indices}

The time course of mean arterial pressure (MAP) is shown in Fig. 1. The administration of E-LPS induced an immediate decrease in MAP, with the maximal hypotensive response being apparent 5 to $10 \mathrm{~min}$ after E-LPS infusion. Although MAP had returned to normal by $1 \mathrm{~h}$ after E-LPS administration, it had declined again by $2 \mathrm{~h}$ after the infusion, indicative of a biphasic response. It subsequently increased gradually, and a hypertensive state was apparent from 4 to $6 \mathrm{~h}$ after infusion. In contrast, MAP remained unchanged until about $3 \mathrm{~h}$ after P-LPS administration, thereafter it increased, and a hypertensive state was maintained until the end of the observation period. Infusion of saline had no effect on MAP in control rats.

Representative M-mode echocardiographic recordings 


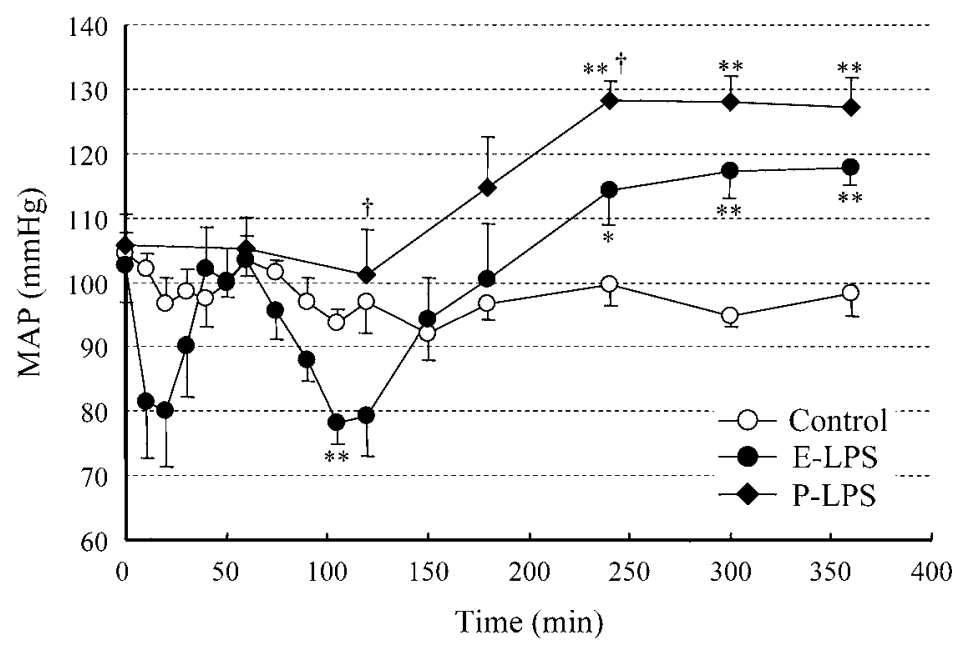

Fig. 1. Time course of mean arterial pressure (MAP) in rats administered ELPS, P-LPS, or physiological saline (control). Data represent means \pm SEM. *: $P<0.05$ and $* *: P<0.01$ vs. Control. ${ }^{\dagger}: P<0.05$ vs. E-LPS.

are shown in Fig. 2, and echocardiographic indices for rats administered E-LPS, P-LPS, or saline are shown in Table 1 and Fig. 3. Both LVDd and LVDs decreased immediately after infusion of E-LPS and remained below the values for control rats throughout the experiment (Table 1). Both IVST and LVPWT were increased 10 min after infusion of E-LPS and remained increased for up to $6 \mathrm{~h}$, although not always significantly (Table 1). LVFS increased from $54.2 \pm 3.5 \%$ at baseline to $77.4 \pm$ 9.9\% at 10 min after E-LPS administration and remained significantly greater than the corresponding values for control rats throughout the observation period (Fig. 3). In contrast, LVDd, IVST, and LVPWT were not significantly affected by P-LPS infusion, whereas LVDs was significantly decreased at 1 and $2 \mathrm{~h}$, but thereafter increased after administration of P-LPS (Table 1). LVFS was increased 1 and $2 \mathrm{~h}$ after infusion of P-LPS but declined thereafter, being significantly reduced compared with that in control rats at $6 \mathrm{~h}$ (Fig. 3). Control rats showed no changes in echocardiographic indices throughout the experiment.

\section{Oxidative stress}

There was no difference in the glutathione redox ratio [reduced (GSH)/oxidized (GSSG)] of the LV myocardium (Fig. 4A) or liver (Fig. 4B) between rats treated with E-LPS, P-LPS, or physiological saline (control) at any of the time points examined.

\section{Plasma concentration of cTnT}

The time course of plasma concentration of cTnT is shown in Fig. 5. The plasma cTnT level was increased significantly at 4 and $7 \mathrm{~h}$ after administration of E-LPS or P-LPS. There was no difference in plasma cTnT level between rats treated with E-LPS and P-LPS. Plasma cTnT was not detected in control rats throughout the experiment.

\section{Plasma concentration of TNF- $\alpha$}

The time course of plasma concentration of TNF- $\alpha$ is shown in Fig. 6. The plasma TNF- $\alpha$ level was increased significantly at 90 min after infusion of E-LPS and declined gradually thereafter. In contrast, the plasma TNF- $\alpha$ level was increased significantly at 30 min after infusion of P-LPS, reached a maximum at 2 $\mathrm{h}$, and then decreased toward control values. The maximal plasma concentration of TNF- $\alpha$ in rats treated with P-LPS was about three times that achieved in rats treated with E-LPS. Control rats showed no change in plasma TNF- $\alpha$ concentration throughout the experiment.

\section{Pathology}

Histological sections of the hearts of rats isolated at $7 \mathrm{~h}$ after administration of E-LPS appeared normal (Fig. 7A), whereas those of the hearts of rats treated with PLPS showed evidence of interstitial edema, congestion, intramyocardial bleeding, myocardial necrosis, infiltra- 


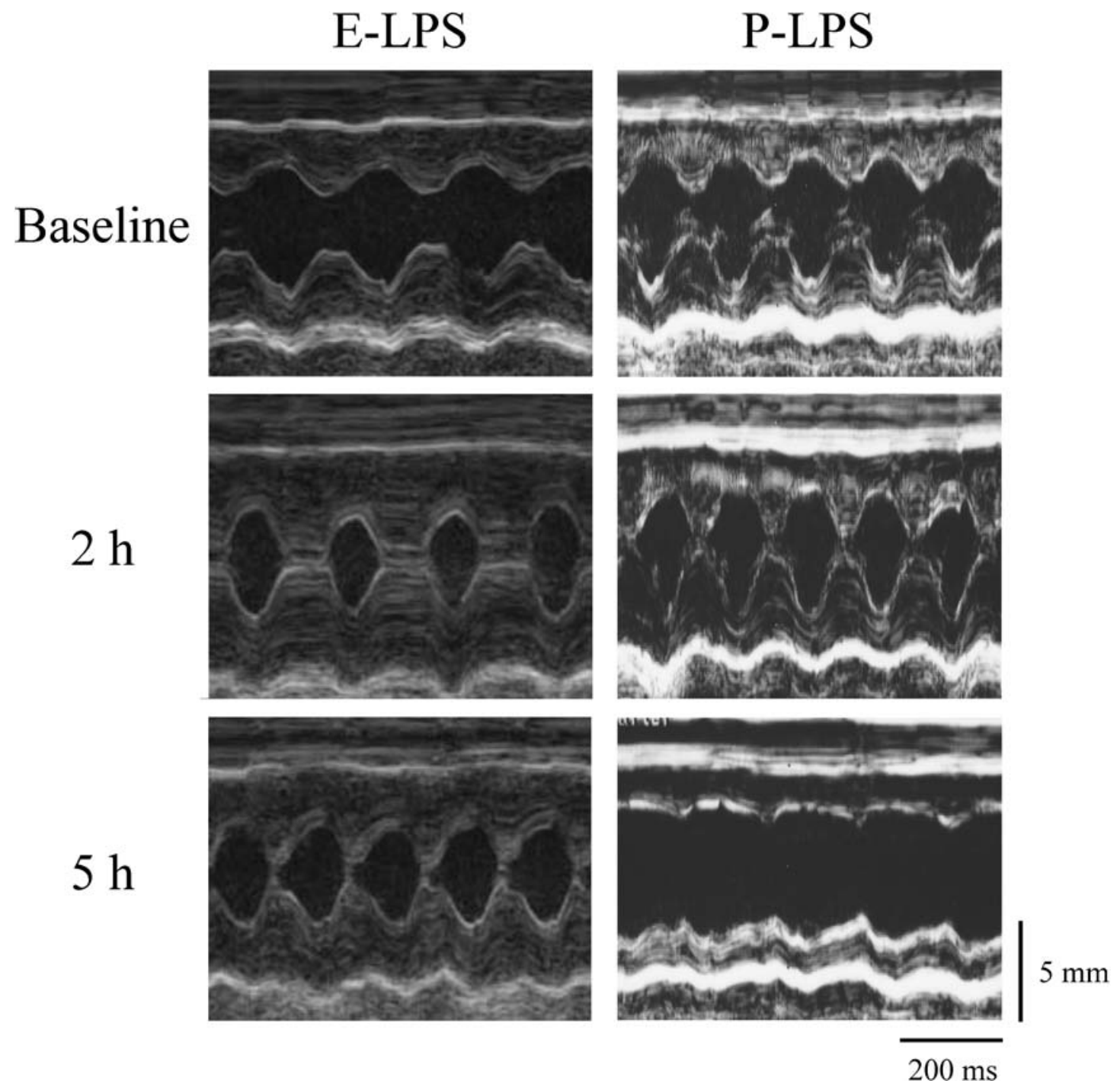

Fig. 2. Representative M-mode echocardiographic recordings obtained from rats at baseline as well as 2 and $5 \mathrm{~h}$ after administration of E-LPS or P-LPS.

tion of inflammatory cells (Fig. 7C), and formation of fibrin thrombi (data not shown). Fibrin thrombi were observed in glomeruli of the kidneys of rats treated with E-LPS (Fig. 7B) or P-LPS (Fig. 7D). No changes were apparent in the heart and kidneys of control rats.

\section{Discussion}

Our results show that the cardiovascular effects of ELPS and P-LPS differ markedly in rats. Thus, the cardiovascular damage induced by LPS appears to depend on the bacterial species from which the endotoxin is derived.

\section{Cardiovascular responses to E-LPS}

The cardiovascular effects induced by infusion of ELPS included a biphasic decrease in MAP, an increase in LV wall thickness, a decrease in LV internal dimensions, and an increase in LVFS. All of these changes were similar to those observed in rats treated with LPS derived from K. pneumoniae in our previous study [13]. However, the changes in arterial pressure, LV internal dimensions, and LV performance induced by E-LPS were less pronounced than those elicited by LPS from K. pneumoniae, even though the dose of E-LPS administered was greater than that of LPS from $K$. pneumoniae, suggesting that the toxicity and potency 
Table 1. Time course of heart rate (HR) and echocardiographic indices in rats after administration of E-LPS, P-LPS, or physiological saline (control)

\begin{tabular}{|c|c|c|c|c|c|c|c|c|}
\hline & Group & Baseline & $1 \mathrm{~h}$ & $2 \mathrm{~h}$ & $3 \mathrm{~h}$ & $4 \mathrm{~h}$ & $5 \mathrm{~h}$ & $6 \mathrm{~h}$ \\
\hline \multirow[t]{3}{*}{ HR (bpm) } & Control & $439 \pm 10$ & $439 \pm 21$ & $424 \pm 25$ & $424 \pm 26$ & $421 \pm 22$ & $427 \pm 23$ & $433 \pm 18$ \\
\hline & E-LPS & $392 \pm 27$ & $401 \pm 23$ & $404 \pm 23$ & $416 \pm 25$ & $396 \pm 12$ & $404 \pm 7$ & $380 \pm 8$ \\
\hline & P-LPS & $387 \pm 23$ & $409 \pm 23$ & $439 \pm 18$ & $423 \pm 23$ & $432 \pm 26$ & $400 \pm 32$ & $381 \pm 39$ \\
\hline \multirow[t]{3}{*}{ LVDd (mm) } & Control & $6.25 \pm 0.10$ & $6.35 \pm 0.09$ & $6.40 \pm 0.12$ & $6.55 \pm 0.22$ & $6.53 \pm 0.09$ & $6.50 \pm 0.12$ & $6.38 \pm 0.20$ \\
\hline & E-LPS & $6.32 \pm 0.16$ & $5.18 \pm 0.40^{*}$ & $4.90 \pm 0.20 * *$ & $5.08 \pm 0.24 * *$ & $5.50 \pm 0.46$ & $5.25 \pm 0.28^{*}$ & $5.05 \pm 0.26^{*}$ \\
\hline & P-LPS & $6.37 \pm 0.12$ & $6.06 \pm 0.25$ & $6.32 \pm 0.16^{\ddagger}$ & $6.53 \pm 0.21^{\ddagger}$ & $6.62 \pm 0.32$ & $6.82 \pm 0.33^{\ddagger}$ & $6.67 \pm 0.36^{\ddagger}$ \\
\hline \multirow[t]{3}{*}{ LVDs (mm) } & Control & $2.98 \pm 0.08$ & $3.05 \pm 0.13$ & $3.05 \pm 0.09$ & $2.90 \pm 0.08$ & $3.15 \pm 0.10$ & $3.08 \pm 0.15$ & $2.95 \pm 0.10$ \\
\hline & E-LPS & $2.90 \pm 0.23$ & $1.40 \pm 0.25^{* *}$ & $0.93 \pm 0.08 * *$ & $1.00 \pm 0.26^{* *}$ & $1.20 \pm 0.31 *$ & $1.15 \pm 0.17 *$ & $1.55 \pm 0.21$ \\
\hline & P-LPS & $2.18 \pm 0.24$ & $1.58 \pm 0.24 *$ & $1.70 \pm 0.31 * *$ & $2.78 \pm 0.53$ & $3.37 \pm 0.75$ & $3.43 \pm 0.84$ & $3.50 \pm 0.86^{*}$ \\
\hline \multirow[t]{3}{*}{ IVST (mm) } & Control & $1.55 \pm 0.03$ & $1.55 \pm 0.03$ & $1.53 \pm 0.05$ & $1.53 \pm 0.05$ & $1.53 \pm 0.05$ & $1.45 \pm 0.03$ & $1.55 \pm 0.06$ \\
\hline & E-LPS & $1.60 \pm 0.04$ & $1.90 \pm 0.17$ & $2.00 \pm 0.11^{* *}$ & $1.80 \pm 0.14$ & $1.88 \pm 0.13^{*}$ & $1.65 \pm 0.05$ & $1.78 \pm 0.06$ \\
\hline & P-LPS & $1.48 \pm 0.08$ & $1.47 \pm 0.08$ & $1.43 \pm 0.06^{\ddagger}$ & $1.47 \pm 0.10$ & $1.38 \pm 0.11^{\ddagger}$ & $1.25 \pm 0.11^{\ddagger}$ & $1.35 \pm 0.12^{\dagger}$ \\
\hline \multirow[t]{3}{*}{ LVPWT (mm) } & Control & $1.58 \pm 0.03$ & $1.55 \pm 0.03$ & $1.60 \pm 0.00$ & $1.55 \pm 0.03$ & $1.58 \pm 0.03$ & $1.53 \pm 0.05$ & $1.63 \pm 0.06$ \\
\hline & E-LPS & $1.60 \pm 0.03$ & $2.07 \pm 0.26$ & $2.12 \pm 0.17^{*}$ & $2.08 \pm 0.19^{*}$ & $2.03 \pm 0.12 *$ & $1.95 \pm 0.12 *$ & $1.88 \pm 0.13$ \\
\hline & P-LPS & $1.48 \pm 0.09$ & $1.50 \pm 0.07$ & $1.42 \pm 0.05^{\ddagger}$ & $1.47 \pm 0.12$ & $1.35 \pm 0.11^{\ddagger}$ & $1.27 \pm 0.11^{\ddagger}$ & $1.32 \pm 0.12^{\ddagger}$ \\
\hline
\end{tabular}

Values are means \pm SEM. LVDd: left ventricular end-diastolic internal dimension, LVDs: left ventricular end-systolic internal dimension, IVST: intraventricular septal wall thickness, LVPWT: left ventricular posterior wall thickness. $*: P<0.05$ and $* *: P<0.01$ vs. Control. $: P<0.05$ and $\ddagger: P<0.01$ vs. E-LPS.

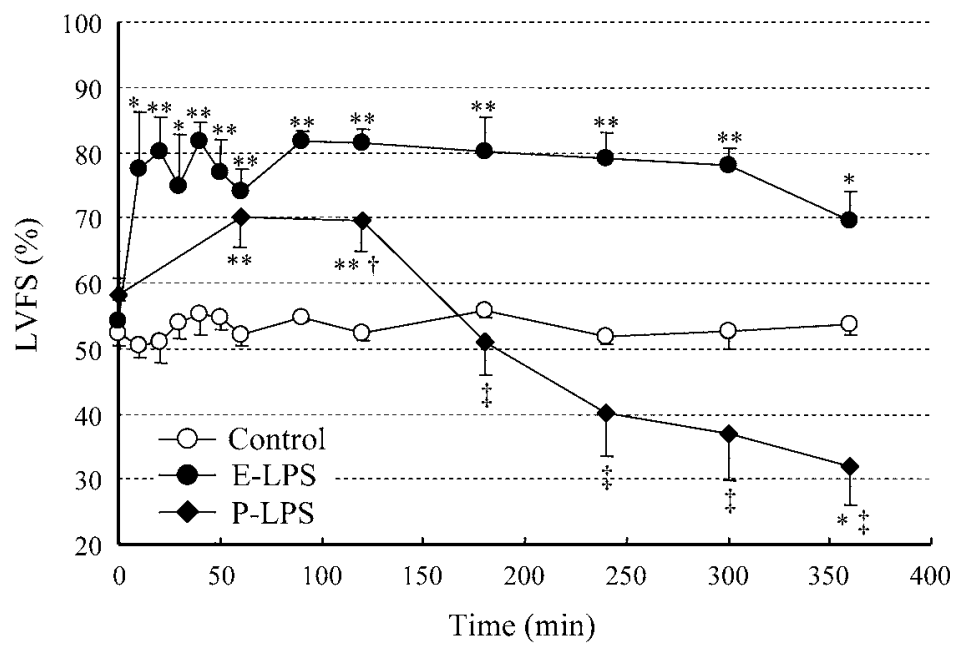

Fig. 3. Time course of left ventricular fractional shortening (LVFS) in rats administered E-LPS, P-LPS, or physiological saline (control). Data represent means \pm SEM. *: $P<0.05$ and $* *: P<0.01$ vs. Control. †: $P<0.05$ and $¥: P<0.01$ vs. E-LPS.

of LPS from $K$. pneumoniae are greater than those of E-LPS. Although the mechanism responsible for the increase in LVFS induced by E-LPS is unclear, it might be attributable to a reduction in LV afterload, given that dehydration due to diarrhea was observed in rats administered E-LPS. Histological analysis did not reveal any abnormalities in the hearts of rats treated with E-LPS, whereas congestion of capillaries and mild edema in the LV myocardium were apparent in rats treated with LPS from K. pneumoniae [13]. It is pos- 

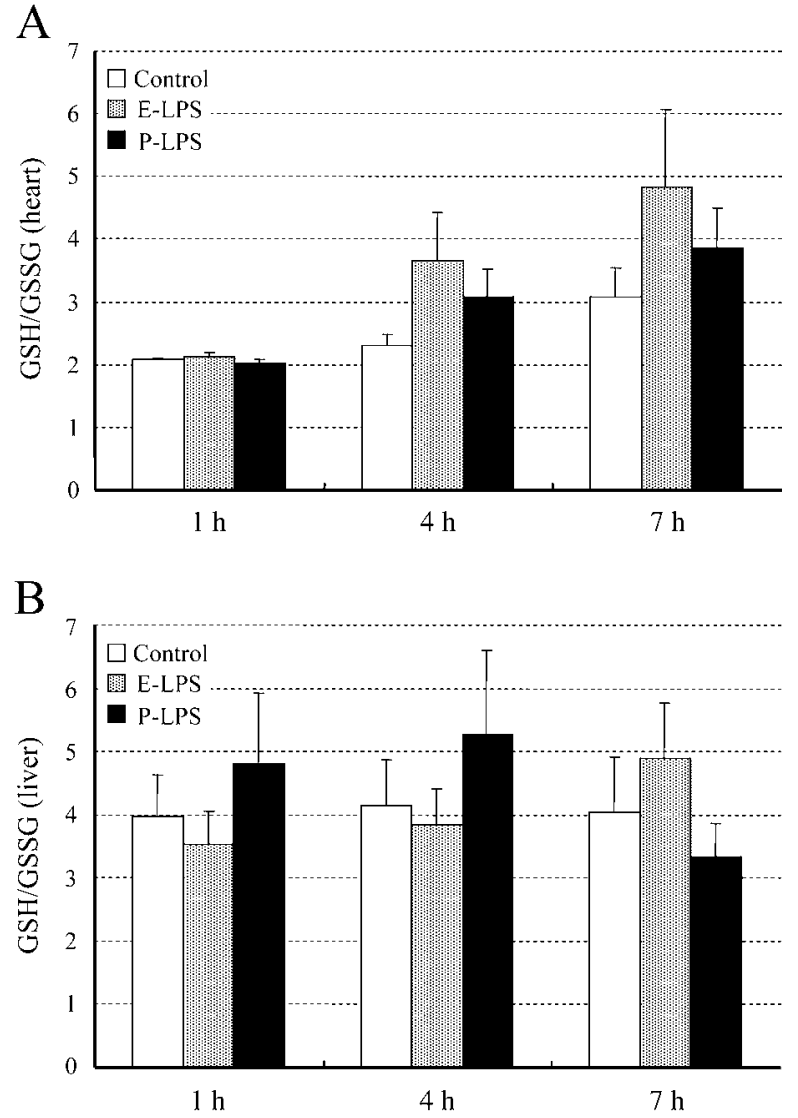

Fig. 4. Glutathione redox ratio (GSH/GSSG) in the heart (A) and liver (B) at 1, 4, and $7 \mathrm{~h}$ after administration of $\mathrm{E}$ LPS, P-LPS, or physiological saline (control). Data represent means \pm SEM.

sible that platelet-activating factor plays a more important role in the cardiovascular effects of LPS from $K$. pneumoniae than in those of E-LPS.

\section{Cardiovascular responses to P-LPS}

The cardiovascular effects of P-LPS included an increase in MAP as well as an initial increase and subsequent decrease in LVFS. In addition, interstitial edema, myocardial necrosis, infiltration of inflammatory cells, and formation of fibrin thrombi were evident in the hearts of rats treated with P-LPS. The impairment in LV function of rats administered P-LPS might be attributable either to the accompanying myocardial necrosis, as revealed by pathological analysis as well as by an increase in the plasma concentration of cTnT, or to $\mathrm{LV}$ afterload mismatch, given that hypertension developed $-3 \mathrm{~h}$ after P-LPS infusion. The production

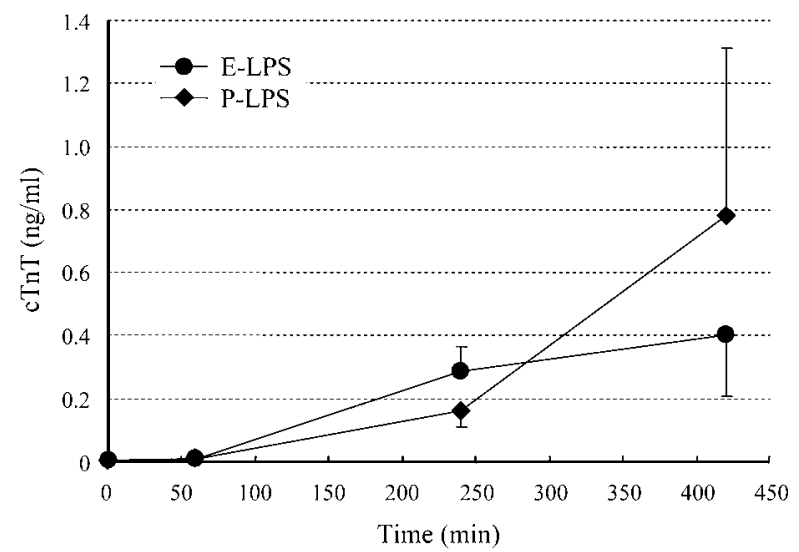

Fig. 5. Time course of plasma cardiac troponin $\mathrm{T}$ (cTnT) concentration in rats administered E-LPS or P-LPS. Data represent means \pm SEM. Plasma cTnT was not detected in control rats throughout the observation period.

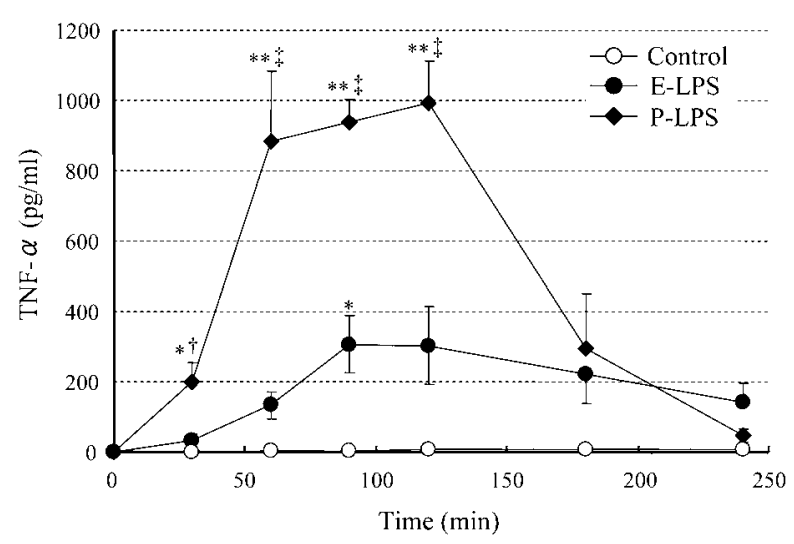

Fig. 6. Time course of plasma tumor necrosis factor- $\alpha$ (TNF- $\alpha$ ) concentration in rats administered E-LPS, P-LPS, or physiological saline (control). Data represent means \pm SEM. *: $P<0.05$ and $* *: P<0.01$ vs. Control. ${ }^{\dagger}: P<0.05$ and $P<0.01$ vs. E-LPS.

of TNF- $\alpha$ induced by LPS administration has been known to elicit hypotension via NO production in endothelium, whereas we observed an increase in MAP as early as $3 \mathrm{~h}$ after administration of P-LPS in the present study. It has been reported that a marked increase in endothelin (ET), a potent vasoconstrictive peptide, was observed after infusion of endotoxin in rats [27]. Although the mechanism of vasoconstriction cannot be explained in our present study, we speculate that an increase in MAP was induced by the effects of a vasopressor substance, such as ET, on vascular smooth 

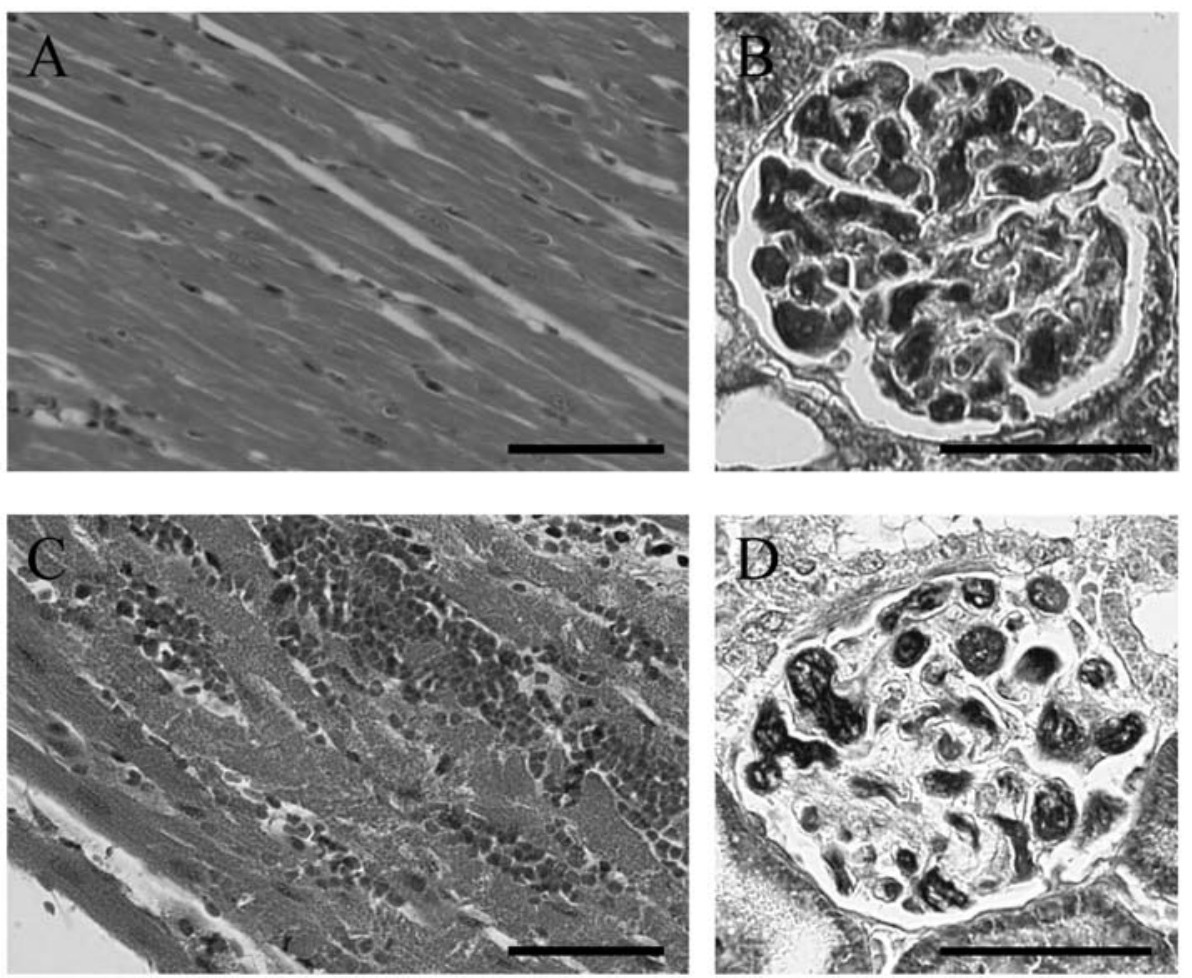

Fig. 7. Representative histological sections of the heart (A, C) and kidney (B, D) of rats at $7 \mathrm{~h}$ after administration of E-LPS (A, B) or P-LPS (C, D). The heart was stained with hematoxylineosin, and the kidney was stained with phosphotungstic acid-hematoxylin. Scale bar $=50 \mu \mathrm{m}$.

muscle in addition to the impairment of endotheliumdependent vasodilation by LPS-induced vascular endothelial dysfunction. TNF- $\alpha$ has also been found to induce a negative inotropic effect, cardiomyocyte apoptosis, and ventricular remodeling [7, 18]. Given that the plasma concentration of TNF- $\alpha$ is increased in patients with heart failure, this cytokine has been proposed as contributing to the progression of this condition through its direct toxic effects on the cardiovascular system [25]. Pathophysiologically relevant peripheral or increased intramyocardial levels of TNF- $\alpha$ are sufficient to recapitulate many aspects of heart failure, including LV dilation, LV dysfunction, and activation of fetal gene expression, in experimental animals [3, 17]. The marked increase in plasma TNF- $\alpha$ level induced by P-LPS administration might contribute to the associated LV dysfunction.

Differential cardiovascular responses to E-LPS and P-LPS

Since the time course of cardiovascular effects induced by E-LPS was drastically different from that by
P-LPS, the time points for measurements of MAP and echocardiography differed between rats administered ELPS and P-LPS. Our preliminary studies indicated that both arterial pressure and LVFS were altered immediately after administration of E-LPS, as evident in this study. In contrast, both parameters remained almost unchanged early after administration of P-LPS, unlike after administration of E-LPS. Therefore, we focused on the cardiovascular effects of P-LPS on a 1-h basis rather than $10 \mathrm{~min}$ basis. We believe that this differential time course of cardiovascular responses between E-LPS and P-LPS is one of the striking findings of our study.

Although LPS from both bacterial species induced similar increases in the plasma concentration of cTnT, the LV functional responses were substantially different. The plasma concentration of TNF- $\alpha$ in rats treated with P-LPS was greater than that in rats treated with ELPS. The increase in the plasma level of cTnT in the absence of any obvious pathological findings in the hearts of rats treated with E-LPS might be due to func- 
tional alterations in the plasma membrane of cardiomyocytes rather than to cardiomyocyte death. Enzyme release from myocardial cells in the absence of cell necrosis has been observed after reversible ischemia in vitro [24]. Furthermore, TNF- $\alpha$ and its mediators produced by granulocytes may increase the permeability of the cardiomyocyte membrane to macromolecules such as troponin without inducing myocyte necrosis [30]. In rats treated with P-LPS, the increase in the plasma level of cTnT might result from myocyte cell death as well as functional changes in the plasma membrane.

Myocardial oxidative stress has been found to increase in association with experimental $[9,10,21]$ or clinical [1, 19] heart failure. We hypothesized that reactive oxygen species produced within the heart might induce contractile dysfunction and pathological changes in rats treated with LPS. However, neither E-LPS nor P-LPS increased oxidative stress in the heart in the present study.

\section{Study limitations}

In this study, there was a substantial difference between the doses of E-LPS and P-LPS. The doses of both E-LPS and P-LPS were initially decided as the same dose $(2 \mathrm{mg} / \mathrm{kg})$ of LPS derived from $K$. pneumoniae that was used in our previous study [13], however, administration of E-LPS at this dose induced no changes in blood pressure and cardiac function as assessed by echocardiography in preliminary experiments. Therefore, the dose of E-LPS $(10 \mathrm{mg} / \mathrm{kg})$ was decided as the minimum dose at which significant changes in blood pressure and cardiac function were induced. Although our findings suggest that the toxicity of P-LPS is more severe than that of E-LPS, further studies are required to examine the dose-response relationship of each LPS.

\section{Conclusions}

E-LPS and P-LPS elicited distinct patterns of functional and structural responses in the rat cardiovascular system. These differential responses may be attributable in part to the difference in the associated increases in the plasma concentration of TNF- $\alpha$. The cardiovascular effects of LPS thus depend on the causative bacteria.

\section{References}

1. Belch, J.J., Bridges, A.B., Scott, N., and Chopra, M. 1991. Oxygen free radicals and congestive heart failure. Br. Heart. J. 65: 245-248.

2. Boillot, A., Massol, J., Maupoil, V., Grelier, R., Bernard, B., Capellier, G., Berthelot, A., and Barale, F. 1997. Myocardial and vascular adrenergic alterations in a rat model of endotoxin shock: reversal by an anti-tumor necrosis factor-alpha monoclonal antibody. Crit. Care. Med. 25: 504-511.

3. Bozkurt, B., Kribbs, S.B., Clubb, F.J. Jr., Michael, L.H., Didenko, V.V., Hornsby, P.J., Seta, Y., Oral, H., Spinale, F.G., and Mann, D.L. 1998. Pathophysiologically relevant concentrations of tumor necrosis factor- $\alpha$ promote progressive left ventricular dysfunction and remodeling in rats. Circulation 97: 1382-1391.

4. Busse, R. and Mulsch, A. 1990. Induction of nitric oxide synthase by cytokines in vascular smooth muscle cells. FEBS. Lett. 275: 87-90.

5. Ferrari, R., Ceconi, C., Curello, S., Alfieri, O., and Visioli, O. 1993. Myocardial damage during ischaemia and reperfusion. Eur. Heart. J. 14: 25-30.

6. Fink, M.P. and Heard, S.O. 1990. Laboratory models of sepsis and septic shock. J. Surg. Res. 49: 186-196.

7. Finkel, M.S., Oddis, C.V., Jacob, T.D., Watkins, S.C., Hattler, B.G., and Simmons, R.L. 1992. Negative inotropic effects of cytokines on the heart mediated by nitric oxide. Science 257: 387-389.

8. Griffith, O.W. 1980. Determination of glutathione and glutathione disulfide using glutathione reductase and 2vinylpyridine. Anal. Biochem. 106: 207-212.

9. Hill, M.F. and Singal, P.K. 1997. Right and left myocardial antioxidant responses during heart failure subsequent to myocardial infarction. Circulation 96: 2414-2420.

10. Ide, T., Tsutsui, H., Kinugawa, S., Utsumi, H., Kang, D., Hattori, N., Uchida, K., Arimura, K., Egashira, K., and Takeshita, A. 1999. Mitochondrial electron transport complex I is the potential source of oxygen free radicals in the failing myocardium. Circ. Res. 85: 357-363.

11. Iwase, M., Kanazawa, H., Kato, Y., Nishizawa, T., Somura, F., Ishiki, R., Nagata, K., Hashimoto, K., Takagi, K., Izawa, H., and Yokota, M. 2004. Growth hormone-releasing peptide can improve left ventricular dysfunction and attenuate dilation in dilated cardiomyopathic hamsters. Cardiovasc. Res. 61: 30-38.

12. Iwase, M., Uechi, M., Vatner, D.E., Asai, K., Shannon, R.P., Kudej, R.K., Wagner, T.E., Wight, D.C., Patrick, T.A., Ishikawa, Y., Homcy, C.J., and Vatner, S.F. 1997. Cardiomyopathy induced by cardiac Gs alpha overexpression. Am. J. Physiol. 272: H585-H589.

13. Iwase, M., Yokota, M., Kitaichi, K., Wang, L., Takagi, K., Nagasaka, T., Izawa, H., and Hasegawa, T. 2001. Cardiac functional and structural alterations induced by endotoxin in rats: importance of platelet-activating factor. Crit. Care. Med. 29: 609-617.

14. Kato, Y., Iwase, M., Kanazawa, H., Kawata, N., Yoshimori, 
Y., Hashimoto, K., Yokoi, T., Noda, A., Takagi, K., Koike, Y., Nishizawa, T., Nishimura, M., and Yokota, M. 2003. Progressive development of pulmonary hypertension leading to right ventricular hypertrophy assessed by echocardiography in rats. Exp. Anim. 52: 285-294.

15. Kato, Y., Iwase, M., Kanazawa, H., Nishizawa, T., Zhao, Y.L., Takagi, K., Nagata, K., Noda, A., Koike, Y., and Yokota, M. 2003. Validity and application of noninvasive measurement of blood pressure in hamsters. Exp. Anim. 52: 359-363.

16. Katus, H.A., Remppis, A., Neumann, F.J., Scheffold, T., Diederich, K.W., Vinar, G., Noe, A., Matern, G., and Kuebler, W. 1991. Diagnostic efficiency of troponin T measurements in acute myocardial infarction. Circulation 83: 902-912.

17. Kubota, T., McTiernan, C.F., and Frye, C.S. 1997. Dilated cardiomyopathy in transgenic mice with cardiac specific overexpression of tumor necrosis factor- $\alpha$. Circ. Res. 81: 627-635.

18. Kubota, T., Miyagishima, M., Frye, C.S., Alber, S.M., Bounoutas, G.S., Kadokami, T., Watkins, S.C., McTiernan, C.F., and Feldman, A.M. 2001. Overexpression of tumor necrosis factor- $\alpha$ activates both anti- and pro-apoptotic pathways in the myocardium. J. Mol. Cell Cardiol. 33: 1331-1344.

19. Mallat, Z., Philip, I., Lebret, M., Chatel, D., Maclouf, J., and Tedgui, A. 1998. Elevated levels of 8-iso-prostaglandin F2 $\alpha$ in pericardial fluid of patients with heart failure: a potential role for in vivo oxidant stress in ventricular dilatation progression to heart failure. Circulation 97: 15361539 .

20. Nathan, C.F., Murray, H.W., and Cohn, Z.A. 1980. The macrophage as an effector cell. N. Engl. J. Med. 303: 622626.

21. Nishizawa, T., Iwase, M., Kanazawa, H., Ichihara, S., Ichihara, G., Nagata, K., Obata, K., Kitaichi, K., Yokoi, T., Watanabe, M., Tsunematsu, T., Ishikawa, Y., Murohara, T., and Yokota, M. 2004. Serial alterations of betaadrenergic signaling in dilated cardiomyopathic hamsters: possible role of myocardial oxidative stress. Circ. J. 68:
1051-1060.

22. Parker, M.M., Shelhamer, J.H., Bacharach, S.L., Green, M.V., Natanson, C., Frederick, T.M., Damske, B.A., and Parrillo, J.E. 1984. Profound but reversible myocardial depression in patients with septic shock. Ann. Intern. Med. 100: 483-490.

23. Parrillo, J.E., Burch, C., Shelhamer, J.H., Parker, M.M., Natanson, C., and Schuette, W. 1985. A circulating myocardial depressant substance in humans with septic shock. Septic shock patients with a reduced ejection fraction have a circulating factor that depresses in vitro myocardial cell performance. J. Clin. Invest. 76: 1539-1553.

24. Piper, H.M., Schwartz, P., Spahr, R., Hutter, J.F., and Spieckermann, P.G. 1984. Early enzyme release from myocardial cells is not due to irreversible cell damage. $J$. Mol. Cell. Cardiol. 16: 385-388.

25. Seta, Y., Shan, K., Bozkurt, B., Oral, H., and Mann, D.L. 1996. Basic mechanisms in heart failure: the cytokine hypothesis. J. Cardiac. Failure 2: 243-249.

26. Singh, N., Dhalla, A.K., Seneviratne, C., and Singal, P.K. 1995. Oxidative stress and heart failure. Mol. Cell Biochem. 147: 77-81.

27. Sugiura, M., Inagami, T., and Kon, V. 1989. Endotoxin stimulates endothelin-release in vivo and in vitro as determined by radioimmunoassay. Biochem. Biophys. Res. Commun. 161: 1220-1227.

28. Tietze, F. 1969. Enzyme method for quantitative determination of nanogram amounts of total and oxidized glutathione: application to mammalian blood and other tissues. Anal. Biochem. 27: 502-522.

29. Wichterman, K.A., Baue, A.E., and Chaudry, I.H. 1980. Sepsis and septic shock-a review of laboratory models and a proposal. J. Surg. Res. 29: 189-201.

30. Wu, A.H. and Ford, L. 1999. Release of cardiac troponin in acute coronary syndromes: ischemia or necrosis? Clin. Chim. Acta 284: 161-174.

31. Xie, J., Joseph, K.O., Bagby, G.J., Giles, T.D., and Greenberg, S.S. 1997. Dissociation of TNF- $\alpha$ from endotoxin-induced nitric oxide and acute-phase hypotension. Am. J. Physiol. 273: H164-H174. 